\title{
APPLICATIONS OF THE EULER CHARACTERISTIC IN BIFURCATION THEORY
}

\author{
SLAWOMIR RYBICKI
}

\begin{abstract}
Let $f: R^{n} \times R^{k} \rightarrow R^{n}$ be a continuous map such that $f(0, \lambda)=0$ for all $\lambda \in R^{k}$. In this article we formulate, in terms of the Euler characteristic of algebraic sets, sufficient conditions for the existence of bifurcation points of the equation $f(x, \lambda)=0$. Moreaver we apply these results in bifurcation theory to ordinary differential equations. It is worth to point out that in the last paragraph we show how to verify, by computer, the assumptions of the theorems of this paper.
\end{abstract}

\section{Introduction}

In [A] Alexander has defined an invariant which nontriviality implies the existence of a bifurcation point of a continuous map $f: R^{n} \times R^{k} \rightarrow R^{n}$ such that $f(0, \lambda)=0$ for all $\lambda \in R^{k}$. This invariant is an element of the group $\pi_{k-1}(G L(n))$. Generally it is difficult to verify if this invariant is a nontrivial element in $\pi_{k-1}(G L(n))$.

Krasnosielski in [K] has proved a 1-parameter bifurcation thcorem which is a very useful tool in bifurcation theory. This theorem gives sufficient conditions for the existence of a bifurcation point of $f$ in the case $k=1$.

Many authors have proved generalizations of the classical Krasnosielski theorem (see [MA], [R1], [R2]).

We are interested in formulating sufficient conditions for the existence of bifurcation points of $f$ in case the dimension of the parameter space is greather than one and when the Alexander invariant can not be applied.

In $[\mathrm{S} 1],[\mathrm{S} 2]$ and $[\mathrm{W}]$ the authors have proved very interesting formulas to a computation of the Euler characteristic of algebraic sets in terms of the Brouwer topological degree of suitable maps.

In the first part of this paper (using these formulas) we formulate and prove sufficient conditions for the existence of bifurcation points of $f$.

Namely, we define (Def. 1.4.) a set of essential maps (Ess $(n, k)$ ) and prove that $0 \in R$ is not an isolated bifurcation point of any essential map $f$ (Prop. 1.1.). 
We also define (Def. 1.3.) a set, of regular maps $(\operatorname{Reg}(n, k))$ and show how to verify if a regular map is an essential map (Prop. 1.3.).

In Proposition 1.4. we formulate sufficient condition for the existence of a bifurcation point of a homogeneous map $f$ (for definition of a homogeneous map see Def. 1.2.).

As the last case we consider a sct of even maps (Even $(n, k)$, Def. 1.5.). In Proposition 1.5. we give sufficient conditions for the existence of a bifurcation point of even map.

Notice that the assumptions of Propositions 1.4., 1.5., 1.6. are expressed in terms of the Brouwer topological degree of polynomial maps. From this point of view it is important to compute the Brouwer topological degree for polynomial maps.

Nierenberg has formulated in $[\mathrm{N}]$ an integral definition of the Brouwer topological degree. We have written a computer progran which computes the topological degree for polynomial maps, in a version given by Nierenberg.

The inportant question is how to verify that $f \in \operatorname{Reg}(n, k)$. In other words we must verify if $0 \in R^{k}$ is an isolated point in $\Psi^{-1}(0)$. There are computer algorithms which allow us to check if $0 \in R^{k}$ is an isolated zero of the polynomial map $\Psi: R^{k} \rightarrow R^{k}$. These algorithms are based on the Eisenbud and Levine results (see [E.L.]). There is a computer program, writen by Andrzej Lecki from University of Gdansk, which is based on such kinds of algorithms. Using this program we can verify if $f \in \operatorname{Reg}(n, k)$.

Acknowledgement. The author wishes to thanks to Andrzej Lecki for several helpful comments.

In the second paragraph we apply topological results of this article to the bifurcation theory for ordinary differential cquations (Th. 2.1., 2.2., 2.3.).

In the last part of this paper we show how to verify by computer the assumptions of the theorems of this paper.

\section{Results}

Denote by $X$ and $Y$ Banach spaces and by $f: X \times R^{k} \rightarrow Y$ a continuous operator such that $f(0, \lambda)=0$ for all $\lambda \in R^{k}$.

Definition 1.1. A point $\lambda_{0} \in R^{k}$ is said to be a bifurcation point of the equation (*) $f(x, \lambda)=0$, if

$$
\left(0, \lambda_{0}\right) \in \text { closure }\left\{(x, \lambda) \in X \times R^{k}: f(x, \lambda)=0 \text { and } x \neq 0\right\} \text {. }
$$

The set of bifurcation points of the equation (*) will be denoted by Bif $(f)$.

Consider a $C^{1}$ map $f: R^{n} \times R^{k} \rightarrow R^{n}$ such that $f(0, \lambda)=0$ for all $\lambda \in R$ and define a map $\Phi: R^{k} \rightarrow R$ by $\Phi(\lambda)=\operatorname{det}\left(D_{x} f(0, \lambda)\right)$.

Definition 1.2. A map $f$ is said to be a homogeneous map, if $\Phi(\lambda)$ is a homogeneous polynomial of degree greather than 1 . 
The set of homogeneous maps will be denoted by Hom $(n, k)$.

Definition 1.3. A map $f: R^{n} \times R^{k} \rightarrow R^{n}$ is said to be a regular map, if

1) $f \in \operatorname{Hom}(n, k)$,

2) $\Phi$ has an isolated critical point at the origin.

The set of regular maps will be denoted by $\operatorname{Reg}(n, k)$.

Define a map $\Psi: R^{k} \rightarrow R^{k}$ by the formula

$$
\Psi(\lambda)=\left(\left[\frac{\partial \Phi}{\partial \lambda_{1}}(\lambda)\right]^{2}+\Phi(\lambda)^{2}, \ldots,\left[\frac{\partial \Phi}{\partial \lambda_{k}}(\lambda)\right]^{2}+\Phi(\lambda)^{2}\right)
$$

and notice that if $\Psi\left(\lambda_{0}\right)=0$, then $\Psi\left(t \cdot \lambda_{0}\right)=0$ for all $t \in R$.

Remark 1.1. The map $\Phi$ has an isolated critical point at the origin iff $\Psi^{-1}(0)=\{0\}$.

Using this remark we will show that a map considered in Example 3.2. is a regular map.

Definition 1.4. A map $f: R^{n} \times R^{k} \rightarrow R^{n}$ is said to be an essential map, if

1) $f \in \operatorname{Reg}(n, k)$,

2) $\Phi^{-1}(0) \neq\{0\}$.

The set of essential maps will be denoted by Ess $(n, k)$.

Proposition 1.1. If $f \in$ Ess $(n, k)$, then $\operatorname{Bif}(f)=\Phi^{-1}(0)$. In particular, Bif $(f) \neq \varnothing$.

Proof: The set $\Phi^{-1}(0)-\{0\}$ is a $(k-1)$-dimensional manifold.

Fix $\lambda_{0} \in \Phi^{-1}(0)-\{0\}$ and 1-dimensional manifold $N\left(\lambda_{0}\right)$ such that manifolds $N\left(\lambda_{0}\right)$ and $\Phi^{-1}(0)-\{0\}$ are transversal at the point $\lambda_{0}$. It is easy to sce that the map $\Phi$ changes sign, at the point $\lambda_{0}$, along $N\left(\lambda_{0}\right)$. So, applying Krasnosielski theorem (see $[\mathrm{K}]$ ) we show that $\lambda_{0} \in \operatorname{Bif}(f)$. The set Bif $(f)$ is closed, that is why $0 \in \operatorname{Bif}(f)$.

Denote by $d \Phi: R^{k} \rightarrow R^{k}$ the gradient of the map $\Phi$.

If $f \in \operatorname{Reg}(n, k)$ then $d \Phi^{-1}(0)=\{0\}$. Denote by $\chi(B)$ the Euler characteristic of the manifold $B$ and by deg $d \Phi$ the Brouwer topological degree of $d \Phi$ with respect to a disc centered at the origin.

Proposition 1.2. If $f \in \operatorname{Ess}(n, k)$, then for any $\varepsilon>0$

i) $\Phi^{-1}(0) \cap S_{\varepsilon}^{k-1}$ is a $(k-2)$-dimensional manifold,

ii) $\chi\left(\Phi^{-1}(0) \cap S_{\varepsilon}^{k-1}\right)=\left(1+(-1)^{k}\right) \cdot(1-\operatorname{deg} d \Phi)$.

Proof: Notice that $\Phi^{-1}(0)-\{0\}$ is a $(k-1)$-dimensional manifold transversal to $S_{\varepsilon}^{k-1}$. That is why $\Phi^{-1}(0) \cap S_{\varepsilon}^{k-1}$ is a $(k-2)$-dimensional manifold.

Let us put the following notations $A_{+}=\left\{\lambda \in S_{\varepsilon}^{k-1}: \Phi(\lambda) \geq 0\right\}, A_{-}=\{\lambda \in$ $\left.S_{\varepsilon}^{k-1}: \Phi(\lambda) \leq 0\right\}, L=\left\{\lambda \in S_{\varepsilon}^{k-1}: \Phi(\lambda)=0\right\}$. 
C.T.C. Wall in [W] has shown that $\chi\left(A_{+}\right)=1+(-1)^{k-1} \cdot \operatorname{deg} d \Phi$ and $\chi\left(A_{-}\right)=1-\operatorname{deg} d \dot{\Phi}$.

It is well known that $\chi(L)=\chi\left(A_{+}\right)+\chi\left(A_{-}\right)-\chi\left(S^{k-1}\right)$.

Using the above formula and C.T.C. Wall results we obtain the thesis.

Proposition 1.3. If $f \in \operatorname{Reg}(n, k)$ and $\left(1+(-1)^{k}\right) \cdot(1-\operatorname{deg} d \Phi) \neq 0$, then $f \in \operatorname{Ess}(n, k)$.

Proof: If $f \in \operatorname{Reg}(n, k)$, then $\Phi^{-1}(0) \cap S_{\epsilon}^{k-1}$ is a $(k-2)$-dimensional manifold or is an empty set. From Proposition 1.2. and from the assumptions it follows that $\Phi^{-1}(0) \cap S_{\varepsilon}^{k-1}$ is not empty set. So our proof is completed.

In this part of the paragraph we formulate sufficient conditions for the existence of bifurcation points, in a case when $f \in \operatorname{Hom}(n, k)$.

Let us denote $B_{i}=\left\{\lambda \in S_{1}^{k-1}:(-1)^{i+1} \cdot \Phi(\lambda) \geq 0\right\}$ for $i=1,2$ and let $m$ be the degree of the polynomial $\Phi(\lambda)$.

If $m$ is an odd number then $\Phi$ has to change a sign near the origin that is why it is enough to consider only the case of even $m$.

Define polynomials $\Gamma_{i}: R^{k} \rightarrow R$ by the formula

$$
\Gamma_{i}(\lambda)=(-1)^{i} \Phi(\lambda)-\|\lambda\|^{m+2},
$$

for $i=1,2$. By $d \Gamma_{i}: R^{k} \rightarrow R^{k}$ we denote the gradient of $\Gamma_{i}$.

By $\operatorname{deg}\left(Q, D_{\varepsilon}^{k}, 0\right)$ we will denote the Brouwer topological degree of the map $Q$ computed on the disc $D_{\varepsilon}^{k}$ with respect to the origin.

Proposition 1.4. There is a positive number $\varepsilon>0$ such that $d \Gamma_{i}^{-1}(0) \cap D_{\varepsilon}^{k}$ $=\{0\}$. If $\operatorname{deg}\left(d \Gamma_{i}, D_{\varepsilon}^{k}, 0\right) \neq(-1)^{k}$ for $i=1,2$, then for any $\varepsilon_{1}>0$ Bif $(f)$ $\cap S_{\varepsilon_{1}}^{k-1} \neq \varnothing$, in particular, $0 \in \mathrm{Bif}(f)$. Moreover the topological dimension of the set Bif $(f) \cap S_{\varepsilon_{1}}^{k-1}$ is equal to $k-2$.

Proof: First of all we will show that $\chi\left(B_{i}\right) \neq \chi\left(S^{k-1}\right)$ for $i=1,2$. Szafraniec has shown in [S2] that there is $\varepsilon>0$ such that $d \Gamma_{i}^{-1}(0) \cap D_{\varepsilon}^{k}=\{0\}$. If $\operatorname{deg}\left(d \Gamma_{i}, D_{\varepsilon}^{k}, 0\right) \neq(-1)^{k}$, then $1-\operatorname{deg}\left(d \Gamma_{2}, D_{\varepsilon}^{k}, 0\right) \neq 1+(-1)^{k-1}$. It is well known fact that $\chi\left(S^{k-1}\right)=1+(-1)^{k-1}$. From Corollary $I$ in [S2] it follows that $\chi\left(B_{i}\right)=\mathrm{I}-\operatorname{deg}\left(d \Gamma_{i}, D_{\varepsilon}^{k}, 0\right)$. So, we have $\chi\left(B_{i}\right) \neq \chi\left(S^{k-1}\right)$. From the above it follows that $B_{i} \neq S^{k-1}$ for $i=1,2$. Fix $\lambda_{i} \in S^{k-1}-B_{i}$ for $i=1,2$. Let $\xi:[0,1] \rightarrow S^{k-1}$ denote a continuous map such that $\xi(0)=\lambda_{1}$ and $\xi(1)=\lambda_{2}$.

Consider a composition $\Phi \circ \xi:[0,1] \rightarrow R$. It is obvious that $(\Phi \circ \xi)\left(\lambda_{1}\right)$. $\cdot(\Phi \circ \xi)\left(\lambda_{2}\right)<0$. So, from Krasnosielski theorem (see [K]) it follows that

$$
\operatorname{Bif}(f) \cap \xi([0,1]) \neq \varnothing .
$$

It is easy to verify that if $\lambda_{0} \in \operatorname{Bif}(f)$, then $t \cdot \lambda_{0} \in \operatorname{Bif}(f)$ for all $t \in R$. So, our proof is completed. 
In the last part of this section we turn to a case when a map $f$ is such that $\Phi(\lambda)$ is not necessary an homogeneous polynomial.

Definition 1.5. A map $f: R^{n} \times R^{k} \rightarrow R^{n}$ is said to be an even map, if $\Phi(\lambda)$ is a polynomial such that $\Phi(-\lambda)=\Phi(\lambda)$.

The set of even maps will be denoted by Even $(n, k)$.

Fix $f \in$ Even $(n, k)$ and denote by $d$ the degreee of $\Phi$. Choose any $\varepsilon>0$. Define a map $\Lambda_{\varepsilon}: D_{\varepsilon}^{k+1} \rightarrow R$ as follows

$$
\Lambda_{\varepsilon}(\lambda)=\Lambda_{\varepsilon}\left(\lambda_{1}, \ldots, \lambda_{k+1}\right)=\|\lambda\|^{d+2} \cdot \Phi\left(\frac{\varepsilon \lambda_{1}}{\|\lambda\|}, \ldots, \frac{\varepsilon \lambda_{k}}{\|\lambda\|}\right) .
$$

Remark 1.2. Notice that $\Lambda_{\varepsilon}(\lambda)$ is a homogeneous polynomial and that $\operatorname{sgn} \Lambda_{\varepsilon}(\lambda)=\operatorname{sgn} \Phi(P(\lambda))$ for $\lambda \in S_{\varepsilon}^{k}$, where $P: S_{\varepsilon}^{k} \rightarrow D_{\varepsilon}^{k}$ is the projection given by the formula $P(\lambda)=\left(\lambda_{1}, \ldots, \lambda_{k}\right)$.

Define polynomials $\sum_{i}^{\varepsilon}: R^{k+1} \rightarrow R$ as follows

$$
\sum_{i}^{\varepsilon}(\lambda)=(-1)^{i} \Lambda_{\varepsilon}(\lambda)-\left(\lambda_{1}^{2}+\cdots+\lambda_{k+1}^{2}\right)^{\left[\frac{d+2}{2}\right]+1}
$$

for $i=1,2$. By $d \sum_{i}^{\varepsilon}: R^{k+1} \rightarrow R^{k+1}$ we will denote the gradient of $\sum_{i}^{\varepsilon}$.

Proposition 1.5. For sufficiently small $\varepsilon_{1}>0$, if $\operatorname{deg}\left(d \sum_{i}^{\varepsilon}, D_{\varepsilon_{1}}^{k+1}, 0\right)$ $\neq(-1)^{k+1}$ for $i=1,2$, then Bif $(f) \cap D_{\varepsilon_{1}}^{k} \neq \varnothing$; moreover the topological dimension of the set $\mathrm{Bif}(f) \cap D_{\varepsilon_{1}}^{k}$ is equal to $k-1$.

A proof of this proposition is a consequence of Proposition 1.4. and Remark 1.2 .

\section{Applications}

In this section we will use the notations of the first section.

Consider a $C^{1}$ map $g: R \times R^{n} \times R^{k} \rightarrow R^{n}$ and assume that $g(t, x, \lambda)$ can be expressed in the form

$$
g(t, x, \lambda)=A(\lambda) x+\varphi(t, x, \lambda)
$$

where

1) $A(\lambda)$ is a $n \times n$-matrix such that $A(0)=\theta$,

2) $\varphi(t, 0, \lambda)=0$ for all $(t, 0, \lambda) \in R \times R^{n} \times R^{k}$,

3) $D_{x} \varphi(t, 0, \lambda)=0$ for all $(t, 0, \lambda) \in R \times R^{n} \times R^{\hbar}$

We are interested in describing the set of bifurcation points of the following boundary value problem

$$
(*) \quad\left\{\begin{array}{l}
\dot{x}(t)=g(t, x(t), \lambda) \\
x(0)=x(1) .
\end{array}\right.
$$


Let us denote $X=\left\{x \in C^{1}([0,1]): x(0)=x(1)\right\}$ and $Y=C^{0}([0,1])$. Define an operator $F: X \times R^{k} \rightarrow Y$ by the formula

$$
F(x(t), \lambda)=L(\lambda)(x(t))-\varphi(t, x(t), \lambda),
$$

where $L(\lambda)(x(t))=\dot{x}(t)-A(\lambda) x(t)$. Notice that zeroes of the operator $f$ are in one-to-one correspondence with solutions of the problem $(*)$.

Let $f: R^{n} \times R^{k} \rightarrow R^{n}$ be the map defined by $f(x, \lambda)=A(\lambda) x$. Now we are in a position to formulate the main theorem of our paper.

Theorem 2.1. If $f \in$ Ess $(n, k)$ and $\varepsilon$ is a sufficiently small positive number, then

1) Bif $(F) \cap D_{\varepsilon}^{k}=\Phi^{-1}(0) \cap D_{\varepsilon}^{k}$,

2) Bif $(F) \cap S_{\varepsilon}^{k-1}$ is a $(k-2)$-dimensional manifold,

3) $\chi\left(\right.$ Bif $\left.(F) \cap S_{\varepsilon}^{k-1}\right)=\chi\left(\Phi^{-1}(0) \cap S_{\varepsilon}^{k-1}\right)=\left(1+(-1)^{k}\right) \cdot(1-\operatorname{deg} d \Phi)$.

Proof:

Notice that $L(0): X \rightarrow Y$ defined by $L(0)(x(t))=\dot{x}(t)$ is a Fredholm operator with Fredholm index 0 and that $X=X_{0} \oplus X_{1}$ and $Y=Y_{0} \oplus Y_{1}$, where

$$
\begin{gathered}
\left.X_{0}=\operatorname{ker} L(0)=R^{n}=\text { subspace of constant functions }\right\}, \\
Y_{0}=R^{n}=\{\text { subspace of constant functions }\}, \\
Y_{1}=\operatorname{im} L(0)=\left\{x \in C^{0}[0,1]: \int_{0}^{1} x(s) d s=0\right\},
\end{gathered}
$$

(see $[\mathrm{M}]$ for more details).

We begin with the Lyapunov-Schmidt reduction.

Let $P_{0}(x)=\int_{0}^{1} x(s) d s$ and $P_{1}(x)=x-\int_{0}^{1} x(s) d s$ denote the projections of $Y$ onto $Y_{0}, Y_{1}$, respectively. Then the equation $F(x, \lambda)=0$ is equivalent to the system of equations

$$
P_{0}\left(F\left(x_{0}+x_{1}, \lambda\right)\right)=0, P_{1}\left(F\left(x_{0}+x_{1}, \lambda\right)\right)=0,
$$

where $x=x_{0}+x_{1}, x_{0} \in X_{0}, x_{1} \in X_{1}$.

Notice that the map $P_{1} \circ F: X_{0} \oplus X_{1} \oplus R^{k} \rightarrow Y_{1}$ is continuously differentiable near $(0,0,0) \in X_{0} \oplus X_{1} \oplus R^{k}, P_{1} \circ F(0,0, \lambda)=0$, and the Fréchet derivative of $P_{1} \circ F$ with respect to $x_{1}$ at $(0,0,0), D_{x_{1}} P_{1} \circ F(0,0,0)$ is an isomorphism of $X_{1}$ onto $Y_{1}$.

Therefore by the implicit function theorem, there is an open neighbourhood $U$ of $(0,0) \in X_{0} \oplus R^{k}$ and $x_{1} \in C^{1}\left(U, X_{1}\right)$ such that the zeros of $F$ near $(0,0,0)$ are given by $\left(x_{0}, x_{1}\left(x_{0}, \lambda\right), \lambda\right)$ for $\left(x_{0}, \lambda\right) \in X_{0} \oplus R^{k}$.

It is easy to see that $x_{1}\left(x_{0}, \lambda\right)=0\left(\left\|x_{0}\right\|\right)$ at $x_{0}=0$, uniformly for $\lambda$ near 0 . 
From the above it follows that zeros of $F$ are in one-to-one correspondence with zeros of a finite dimensional map $Q: U \rightarrow Y_{0}$ defined by the formula $Q\left(x_{0}, \lambda\right)=\left(P_{0} \circ F\right)\left(x_{0}+x_{1}\left(x_{0}, \lambda\right), \lambda\right)$.

It is not difficult to verify that $Q\left(x_{0}, \lambda\right)$ is a map of the form

$$
Q\left(x_{0}, \lambda\right)=-A(\lambda) x_{0}+\Psi\left(x_{0}, \lambda\right),
$$

where $\Psi(0, \lambda)=0$ and $D_{x_{0}} \Psi(0, \lambda)=0$. Notice that $Q \in \operatorname{Ess}(n, k)$.

The rest is a consequence of Propositions 1.1. and 1.2.

The next theorems give sufficient conditions for the existence of bifurcation points of the operator $F$ for more general class maps than the class Ess $(n, k)$.

Theorem 2.2. If $f \in \operatorname{Hom}(n, k)$ and $\operatorname{deg}\left(d \Gamma_{i}, D_{\varepsilon}^{k}, 0\right) \neq(-1)^{k}$ for $i=1,2$, then for any $\varepsilon_{1}<\varepsilon$ Bif $(F) \cap S_{\varepsilon_{1}}^{k-1} \neq \varnothing$. In particular, $0 \in \operatorname{Bif}(F)$. Moreover the topological dimension of the set $\mathrm{Bif}(F) \cap S_{\varepsilon_{1}}^{k-1}$ is equal to $k-2$.

This theorem is a consequence of Proposition 1.4. A proof of this theorem is similar to the proof of Theorem 2.1.

Theorem 2.3. Let $f \in$ Even $(n, k)$. Then for sufficiently small $\varepsilon$ if $\operatorname{deg}\left(d \sum_{i}^{\varepsilon}, D_{\varepsilon}^{k+1}, 0\right) \neq(-1)^{k+1}$ for $i=1,2$, then Bif $(F) \cap D_{\varepsilon}^{k} \neq \varnothing$. Moreover the topological dimension of the set $\operatorname{Bif}(F) \cap D_{\varepsilon}^{k}$ is equal to $k-1$; in particular, $0 \in \operatorname{Bif}(f)$.

This theorem is a consequence of Proposition 1.5. A proof of this theorem is similar to the proof of Theorem 2.1.

\section{Examples}

We shall remind well known results from singularity theory (see [E.L.]).

Let $\theta_{n}$ be the ring of germs of real analytic functions at $0 \in R^{n}$. If $g_{1}, \ldots, g_{n}$ $\in \theta_{n}$, let us denote by $\left\langle g_{1}, \ldots, g_{n}\right\rangle$ the ideal in $\theta_{n}$ generated by the elements $g_{1}, \ldots, g_{n}$.

For $g=\left(g_{1}, \ldots, g_{n}\right):\left(R^{n}, 0\right) \rightarrow\left(R^{n}, 0\right)$ we put

$$
Q(g)=\theta_{n} /\left\langle g_{1}, \ldots, g_{n}\right\rangle
$$

If $g$ is finite, in the sense that $Q(g)$ is finitc dimensional real vector space, then $0 \in R^{n}$ is isolated in $g^{-1}(0)$.

Consider the following boundary value problem

$$
(* *) \quad\left\{\begin{array}{l}
\dot{x}(t)=g(t, x(t), \lambda), \\
x(0)=x(1)
\end{array}\right.
$$


and assume that the map $g$ satisfies all the assumptions of the previous paragraph. So we can express $g$ in the form $g(t, x, \lambda)=A(\lambda) x+\varphi(t, x, \lambda)$. Theorems 2.1., 2.2., 2.3. show that it is enough to examine only the matrix $A(\lambda)$.

Example 3.1. Assume that $n=k=3$ and that the matrix $A(\lambda)$ is of the form

$$
A(\lambda)=\left[\begin{array}{ccc}
\lambda_{1}+\lambda_{2}, & \lambda_{2}+\lambda_{3}, & \lambda_{1}+\lambda_{3} \\
\lambda_{1}+\lambda_{2}+\lambda_{3}, & \lambda_{1}-\lambda_{2}, & 2 \cdot \lambda_{1}-3 \cdot \lambda_{3} \\
\lambda_{3}, & \lambda_{1}, & 0
\end{array}\right]
$$

Using a computcr program we show that $\operatorname{deg}\left(d \Gamma_{i}, D_{\varepsilon}^{3}, 0\right)=1$ for $i=1,2$. From this it follows that $\operatorname{deg}\left(d \Gamma_{i}, D_{\varepsilon}^{3}, 0\right) \neq(-1)^{3}$, so the assumption of Theorem 2.2. is fulfilled. Applying Theorem 2.2 we claim that there exists $\varepsilon>0$ such that for any $\varepsilon_{1}<\varepsilon$ the intersection of the set of bifurcation points of the problem (**) with $S_{\varepsilon_{1}}^{2}$ is not empty, in particular $0 \in$ Bif $(F)$. Moreover the topological dimension of this intersection is equal to 1 .

Example 3.2. Assume that $n=k=2$ and that the matrix $A(\lambda)$ is of the form

$$
A(\lambda)=\left[\begin{array}{cc}
\lambda_{1}-\lambda_{2}, & \lambda_{1}^{2}-\lambda_{2}^{2} \\
-\lambda_{1}+2 \cdot \lambda_{2}, & \lambda_{1} \cdot \lambda_{2}-\lambda_{2}^{2}
\end{array}\right] .
$$

Define a map $f: R^{2} \times R^{2} \rightarrow R^{2}$ by $f(x, \lambda)=A(\lambda) x$.

We will show that $f \in$ Ess $(2,2)$. Using a computer program we show that $\operatorname{dim} Q(\Psi)=16$, so from the results from singularity thcory it follows that $\Psi^{-1}(0)=\{0\}$, that is why $f \in \operatorname{Reg}(2,2)$.

Computing the Brouwer topological degree (by computer) we obtain deg $d \Phi$ $=-2$ and consequently

$$
\chi\left(\Phi^{-1}(0) \cap S_{\varepsilon}^{1}\right)=\left(1+(-1)^{k}\right) \cdot(1-\operatorname{deg} d \Phi)=\left(1+(-1)^{2}\right) \cdot(1-(-2))=6
$$

From this it follows that $\Phi^{-1}(0) \neq\{0\}$, so $f \in \operatorname{Ess}(2,2)$.

So from Theorem 2.1 it follows that Bif $(F) \cap D_{\varepsilon}^{2}$ consist of exactly six intervals, which emanate from the origin.

\section{References}

[A] J.C. AlexANDER, Bifurcation of zeros of parametrized functions, $J$. Funct. Analy. 29 (1978), 37-53.

[E.L.] D. Eisenbld, H.I. Levine, An algebraic formula for the degree of a $C^{\infty}$ map germ, Ann. Math. 106 (1977), 19-44.

[I.R.1] M. IzYDOREK, S. RYBiCKI, On the number of bifurcation branches of $C^{2}$-maps, accepted for publication in JMAA (1991).

[I.R.2] M. IZYDOREK, S. RYBICKI, On the number of branches of bifurcation points, to appear. 
[K] M.A. KRASNosielski, "Topological methods in the theory of nonlinear integral equations," Pergamon, 1964.

[M] J. MAWHIN, "Topological degree methods in nonlinear boundary value problems," Conference board of the mathematical sciences, Regional conference series in mathematics 40, Amcrican Mathematical Society, Providence, Rhode Island, 1979.

[MA] A. Marino, "La bifurcazione nel caso variazionale," Conf. Sem. Mat. Univ. Bari 132, 14 pp. MR50, 1068, 1973.

[N] L. NierLnberg, "Topics in nonlinear analysis, N.Y.Y.," Lecture Notes, 1974.

[R1] P. RABINOwITz, Some aspects of nonlinear eigenvalue problems, Rocky Moun. J. of Math. 3 (1973), 161-202.

[R2] R. RABINOWITZ, A bifurcation theorem for potential operators, $J$. Funct. Analy. 26 (1977), 48-67.

[S1] Z. SZAFRANIEC, On the Euler characteristic of analytic and algebraic sets, Topology 25, 4 (1986), 411-414.

[S2] Z. SZAFRANIEC, The Euler characteristic of algebraic complete intersections, J. Reine Angew. Math. 397 (1989), 194-201.

[W] C.T.C. WALL, Topological invariance of the Milnor number mod 2, Topology 22 (1989), 345-350.

\author{
Department of Mathematics \\ Technical University of Gdansk \\ ul. Majakowskiego 11/12 \\ 80-952 Gdansk \\ POLAND
}

Primera versió rebuda el 2 d'Octubre de 1990 , darrera versió rebuda el 3 de Juny de 1991 